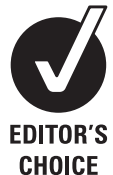

CHOICE

\title{
Intraoperative analysis of sentinel lymph nodes in breast cancer by one-step nucleic acid amplification
}

\author{
Gábor Cserni
}

\section{Correspondence to} Gábor Cserni, University of Szeged, Faculty of Medicine, Department of Pathology, Allomás u. 2., H-6720 Szeged, Hungary and Bács-Kiskun County Teaching Hospital, Nyiri ut 38., H-6000 Kecskemét, Hungary; cserni@freemail.hu

Accepted 7 August 2011 Published Online First 16 November 2011

\begin{abstract}
One-step nucleic acid amplification (OSNA) is a novel method introduced for the lymph node staging of breast cancer and has been tested in multiple series. The present review summarises current literature and concerns related to the new method. The results of this automated molecular assay based on the quantification of cytokeratin 19 mRNA show a $96 \%$ concordance rate with detailed histopathology complemented with immunohistochemistry when alternative slices of the same lymph node are used for the two tests. The low false-negative rate makes OSNA suitable for the intraoperative evaluation of sentinel lymph nodes. The false-positive rate also seems very low. Most discordant cases are explainable by low volume metastases (micrometastases), which may be missing from the material submitted for one test, but not from the different part used for the other test. It is tempting to change the gold standard for comparisons between the methods, and if this is done, histology seems to come out as a weaker test for the identification of metastases. OSNA detects more low volume nodal involvement, but it is uncertain whether these require further axillary treatment, and this will be a subject for future investigations. Therefore, it is also uncertain whether the advantage of OSNA of detecting practically all metastases due to complete sampling of lymph node tissue is clinically more important than the exclusion of metastases greater than micrometastasis that can be reliably done by intraoperative microscopy followed by permanent section histology.
\end{abstract}

Sentinel lymph node (SLN) biopsy is currently the recommended procedure for axillary staging of early breast cancer. ${ }^{1}$ It allows a selective treatment of the axilla, limiting this to patients with involved SLNs.

Intraoperative assessment of the SLN was first introduced to confirm that the blue-stained piece of tissue removed during lymphatic mapping was really a lymph node, ${ }^{2}$ but this indication was soon replaced by the need to know the metastatic status of the SLN during the operation. Therefore the classic nodal staging scenario includes intraoperative microscopy-based pathological assessment of the SLN (frozen sections, touch imprints, scrapes or a combination of these) followed by formalin fixation and paraffin embedding of the remaining tissues and permanent sections used for a final pathological diagnosis of the nodes. This procedure allows the identification of most metastases during the operation, and therefore makes completion axillary lymph node dissection (ALND) possible at the same time, but leaves at least some metastases undisclosed. Micrometastases are espe- cially prone to remain occult during intraoperative assessment. ${ }^{3} 4$ It ensues that the sensitivity of intraoperative pathology is less than optimal, despite a very good specificity of approximately 99-100\%. A meta-analysis of 31 studies on imprint cytology suggested an overall sensitivity of $63 \%$; the pooled sensitivity for macrometastases was higher (81\%) than that for micrometastases $(22 \%){ }^{3}$ A similar meta-analysis of 47 series of intraoperative frozen sections reported a pooled sensitivity of $78 \%$; again sensitivity for macrometastases (94\%) was higher than that for micrometastases $(40 \%){ }^{4}$

To improve on this, several initiatives were tested. Multilevel serial frozen sectioning, ${ }^{5}$ rapid immunohistochemistry applicable with both intraoperative cytology specimens ${ }^{6} 7$ and frozen sections ${ }^{8-10}$ have all resulted in increased sensitivity and time requirement. As an alternative way of intraoperative SLN investigation, molecular assays have also been introduced. ${ }^{11} 12$ Of the two most tested assays, only the one-step nucleic acid amplification (OSNA)-based assay is currently available, whereas the dual (cytokeratin 19 (CK19) and mammaglobin messenger RNA detecting) quantitative reverse transcription (RT) PCR-based assay (BLN assay; Veridex, Raritan, New Jersey, USA) has been withdrawn. This review summarises the methodology of OSNA, the results available at present, their clinical value, and areas of concern with this method in the treatment of breast cancer.

\section{OSNA FOR THE DETECTION OF METASTASIS IN SLN FROM BREAST CANCER PATIENTS}

OSNA for CK19 is currently an automated molecular assay developed by Sysmex, Kobe, Japan. It requires the homogenisation of lymph node tissue in a specific lysis buffer and uses the supernatant (lysate) of the tissues after brief centrifugation. In contrast to RT-PCR, it does not need the extraction or purification of mRNA. It uses RT loopmediated isothermic amplification described by Notomi and colleagues ${ }^{13}$ with a standardised kit (Lynoamp; Sysmex, Kobe, Japan) and a gene amplification detector manufactured by Sysmex, the RD-100i system. ${ }^{12}$ The six primers (including the forward and reverse loop primers) used for the amplification were specially designed to avoid cross-amplification with the two known CK19 pseudogene products. ${ }^{12}$ The isothermic constant temperature of $65^{\circ} \mathrm{C}$ prevents simultaneous genomic DNA amplification. The principle of quantification is the detection of a byproduct of the amplification process (which therefore follows the amplification in quantity): magnesium 
pyrophosphate is characterised by low solubility and fast precipitation after saturation of its aqueous solution. The precipitate results in turbidity that is detected by real-time monitoring in the $\mathrm{RD}-100 \mathrm{i}$. The system is generally run with adequate controls ( $\beta$-actin mRNA, to check mRNA quality; a positive control with given number of copies of CK19 mRNA and a negative control without CK19 mRNA) to allow calibration and check for reagent or instrument matters and contamination issues, respectively. The results are output automatically in a semiquantitative way: - for CK19 mRNA copy numbers less than $250 / \mu 1$, + for copy numbers between 250 and $5000 / \mu 1$ and ++ for copy numbers greater than $5000 / \mu \mathrm{l}$. The lower level positivity $(+)$ has been calculated to reflect micrometastatic lymph node involvement, whereas the ++ result has been proposed to reflect macrometastatic nodal involvement. ${ }^{12} 14$

OSNA can evaluate a maximum of four SLN samples at the same time with an increase in the total time required to obtain the results. A sample should be $0.6 \mathrm{~g}$ or less, therefore larger SLN need to be processed in multiple samples.

Although OSNA is used in the intraoperative setting it may also be viewed as an alternative to permanent section histology, as this is generally the method with which it is compared in validation series. OSNA differs in several aspects from the standard nodal staging procedure based on histology with or without immunohistochemistry (table 1). Some of these differences constitute real advantages, others may reflect disadvantages.

\section{Published validation series for OSNA}

Pubmed was searched for all items published in English for the terms OSNA and breast and sentinel, to the end of June 2011. In addition, a paper peer reviewed by the author and accepted for publication was also included. ${ }^{14}$

All studies used a similar approach, with histology representing the gold standard for comparisons. As it is well understood that the tissue used for the molecular assays cannot be used for histology, and on the other hand, formalin-fixed and paraffin-embedded tissue required for permanent sections is not suitable for quantitative mRNA measurements, lymph node tissue has been divided into pieces with approximately half of the lymph node volume subjected to one test and the other half used for the other. It is well known that halving a lymph node may result in discrepant results simply on the basis of the spatial distribution of the metastasis present in one half and missing from the other. ${ }^{20}$ Metastases are generally non-randomly distributed in the lymph nodes, but are located close to the junction of the tumour-draining afferent lymphatic. ${ }^{21} 22$ Sampling bias arising from using different parts of the lymph nodes for different diagnostic tests has been recognised as a major source of discrepant results. ${ }^{23}$ This is why instead of using two different half nodes for the two different tests, all studies summarised in table 2 used multislicing of the nodes and alternating 1-2 $\mathrm{mm}$ thick slices were submitted for histology and OSNA.

Concordant results by histology and the molecular test require no extra investigations, but the validation studies all analysed discordant results of the two tests further. Discordant case investigation included alternative molecular tests of the lysate stored at $-80^{\circ} \mathrm{C}$, complemented in some studies with further histological analysis (further sectioning and/or immunohistochemistry of the remaining parts of the tissue blocks when this was not initially done). ${ }^{14}{ }^{18}{ }^{24-28}$ Further molecular analysis of the lysate included western blotting for CK19 to demonstrate the presence of the protein in the sample, ${ }^{12} 141824-28$ repeat OSNA to check possible technical failures, ${ }^{27}$ and quantitative RT-PCR after the extraction of total RNA. ${ }^{2} \quad \begin{array}{lllll}14 & 18 & 24 & 27 & 28\end{array}$ The markers used for the quantitative RT-PCR assay were CK19, ${ }^{12}$ or CK19 combined with SAM pointed domain containing Ets transcription factor and forkhead box A1; ${ }^{14} 18242527$ all of these have been selected as breast cancer-specific mRNA markers from 45 candidates during the development of the OSNA method, because all three demonstrated a good discrimination between histologically negative and positive lymph nodes. ${ }^{12}$ Discordant cases that showed western blot or quantitative RT-PCR results consistent with the initial OSNA results (either both negative or both positive) were interpreted as probably due to tissue allocation bias (TAB), and were excluded from the analysis of statistical parameters listed in table 2. Only TAB cases were removed, as they most probably represent a virtual error due to the testing of different areas; possible sample mixups, pathology misinterpretations or changes were not excluded. ${ }^{27}$ It must also be mentioned that TAB cases, by strict

Table 1 Comparison of OSNA and histology for the nodal staging of breast cancer

\begin{tabular}{|c|c|c|}
\hline & Histology & OSNA \\
\hline General & $\begin{array}{l}\text { Complex but subjective evaluation that gives more } \\
\text { information than nodal staging, can detect other nodal } \\
\text { lesions (lymphoma, leukaemic infiltration, capsular nevi, } \\
\text { epithelial inclusions, specific lymphadenitis types such } \\
\text { as toxoplasmosis and tuberculosis in the authors experience) }\end{array}$ & Indirectly detects CK19, and can only be used for staging \\
\hline Morphology & $\begin{array}{l}\text { This is of prime importance in identifying a metastasis } \\
\text { and distinguishing it from other possible mimics }\end{array}$ & None, unless tissue is divided for OSNA and microscopy \\
\hline Quantification & $\begin{array}{l}\text { Generally based on the largest dimension of two-dimensional } \\
\text { evaluation, sometimes complemented by estimations based } \\
\text { on the third dimension (step sectioning) }\end{array}$ & $\begin{array}{l}\text { Quantification of CK19 mRNA RT-LAMP reaction by-product } \\
\text { reflecting tumour burden (volume) }\end{array}$ \\
\hline Staging & $\begin{array}{l}\text { Staging categories such as minimal nodal involvement belonging } \\
\text { to the ITC category, micrometastasis and (macro)metastasis are } \\
\text { defined on the basis of microscopic size and cell numbers, }{ }^{15}{ }_{16} \\
\text { but are less than optimally reproducible }\end{array}$ & $\begin{array}{l}\text { Not suitable for measurements, but a semiquantitative display of } \\
\text { metastatic volume-related results more or less matching the arbitrary } \\
\text { categories of micrometastasis and (macro)metastasis. }\end{array}$ \\
\hline Sampling & $\begin{array}{l}\text { Variable, depending on the distance between cut surfaces produced } \\
\text { for microscopy; the interval tissue is discarded }\end{array}$ & The tissue used for OSNA is completely investigated \\
\hline Protocol & Variable from department to department ${ }^{17}$ & Standardised \\
\hline Time requirement & $\begin{array}{l}\text { A day or more for permanent sections, but only } 10-20 \text { min for } \\
\text { imprint cytology, and a frozen section; could be similar to OSNA } \\
\text { when combined with rapid immunohistochemistry }{ }^{7} \text { or when doing } \\
\text { a labour-intensive frozen sectioning protocol }{ }^{5}\end{array}$ & $\begin{array}{l}\text { Minimum and maximum reported are } 22^{18} \text { and } 116^{19} \mathrm{~min} \text {, respectively. } \\
\text { Mean or median times reported are between } 32 \text { and } 62 \mathrm{~min} \text {, depending } \\
\text { on the number of samples }\end{array}$ \\
\hline
\end{tabular}


Table 2 Results of histological and OSNA tests in different validation series, histology being the gold standard for comparison

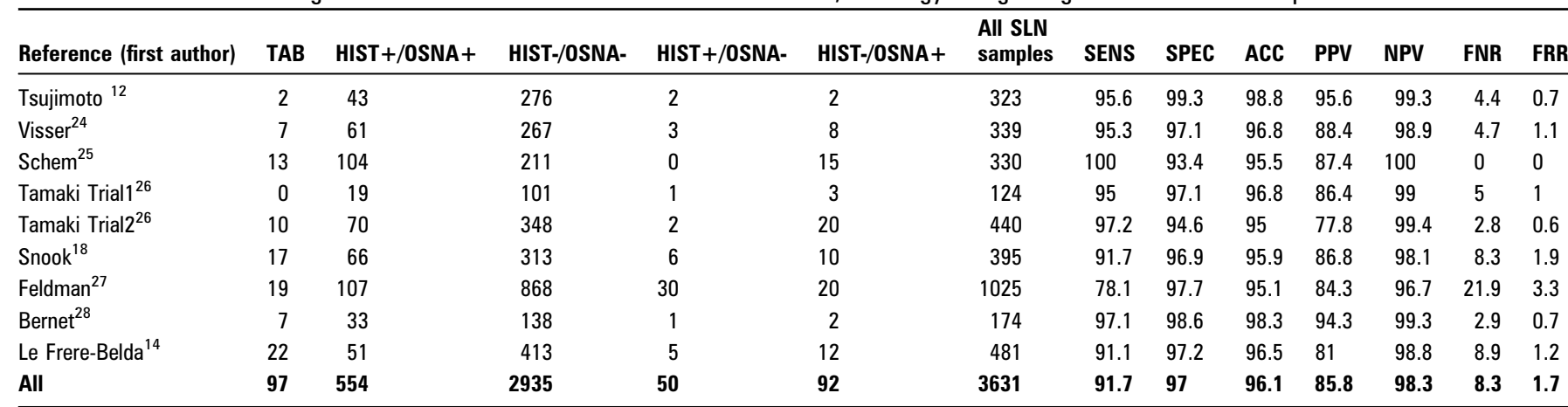

Case numbers represent lymph node samples, which is slightly higher than the lymph node number, as larger nodes had to be assayed as multiple samples.

+ , positive (including micrometastases/macrometastases for histology and $+/++$ results for OSNA); - , negative (including isoltaed tumour cells and copy numbers $<250 / \mu 1$ of lysate, respectively); ACC, accuracy (concordance rate of the two tests); FNR, false-negative rate (false-negatives/all positives); FRR, false-reassurance rate (false-negatives/all testing negative); HIST, histological; NPV, negative predictive value; OSNA, one-step nucleic acid amplification; PPV, positive predictive value; SENS, sensitivity; SPEC, specificity; TAB, tissue allocation bias (samples excluded from analysis). Values from SENS to FRR are expressed as percentages.

definition of a test-to-test comparison, would compose a subset of the false-positive cases, but as other methods are supportive of a true positive OSNA result, it is more realistic to exclude such $T A B$ cases from the analysis, and this has been done in all validation series.

Most studies have clear data presentation and interpretation, but one multi-institutional study had obscure areas in the results section; ${ }^{28}$ for example, the authors stated 'four cases were negative OSNA/positive histology (one macro-metastasis and two micro-metastases)'. The definition of TAB is also less clear in this study and the work-up included CK19 western blotting and PCR as the authors state, although this latter was most probably quantitative RT-PCR. For that study, the summary given in the discussion, mentioning seven cases corresponding to $\mathrm{TAB}$ was considered for table 2 .

All comparative studies to date are supportive of OSNA as a method of metastasis detection in (sentinel) lymph nodes of breast cancer patients. Some of them were performed on axillary lymph nodes without selection, ${ }^{12}{ }^{24-26}$ whereas others used SLNs. ${ }^{14} 182728$ The overall concordance rate between histology and OSNA with alternate tissue slices tested is as high as $96 \%$. Of the discrepant results, $40 \%$ have been attributed to TAB, but alternative slice testing cannot be excluded as a cause in another significant proportion of these discordances. Indeed, several mismatching results showed very low volume nodal involvement, either by histology (small micrometastasis or negative but harbouring isolated tumour cells; ITC) or by OSNA (close to the positivity cut-off value of 250 copies/ $\mu$ l). In fact, such low volume metastases were frequently the sources of mismatching results (table 3). After the exclusion of the study by Tamaki and co-workers, ${ }^{26}$ which does not provide a semiquantitative divi- sion of OSNA-positive cases, it can be established that of the 203 initially mismatching results $142(70 \%)$ were due to cases diagnosed as micrometastasis (41) or labelled as OSNA + (101), and this is well over the cases explained by TAB (87) in these series. Not surprisingly, OSNA identified more low volume nodal involvement than histology.

Importantly, the false-negative rate of the OSNA assay compared with intensive histology was within the generally tolerated $10 \%$ limit $(8.3 \%)$, and overall it is suggested that negative OSNA results give a very low false-reassurance rate of $1.7 \%$ (table 2 ); ie, a negative test not mandating completion ALND is unlikely to be false negative, the negative predictive value being high (98.3\%). A rare cause of false negativity may stem from a few breast cancers being CK19 negative, ${ }^{26}$ although the lack of CK19 immunostaining of the primary tumour might not obviously translate into the lack of CK19 mRNA expression. CK19 is reported to be positive in $98.2 \%$ of breast carcinomas, ${ }^{29} 30$ and testing for CK19 by immunohistochemistry of the primary tumour may warn about this rare phenotype.

As concerns the false-positive results, they are very difficult to assess. By definition and comparison woth the gold standard of detailed histopathology, 92 of 3631 cases (2.5\%) tested positive by OSNA without evidence of nodal involvement at microscopy after the elimination of discordant cases attributed to TAB, whereas 554 of 3631 (15.3\%) samples were true positive by OSNA at the same time (table 2). This proportion of falsepositive tests is reasonably low. Most cases (52/69; 75\% of those that could be evaluated) belonged to the low volume positivity (OSNA+), which has the potential of not being represented in histology sections or being represented only as ITC, as was seen in a few patients. Other rare causes of such mismatches may

Table 3 Micrometastases detected by histology and/or OSNA including the TAB cases

\begin{tabular}{|c|c|c|c|c|c|c|c|c|c|}
\hline $\begin{array}{l}\text { Reference } \\
\text { (first author) }\end{array}$ & TAB & $\begin{array}{l}\text { MIC or } \\
\text { OSNA+ }\end{array}$ & MIC & OSNA+ & $\begin{array}{l}\text { Concordant MIC } \\
\text { and OSNA+ }\end{array}$ & $\begin{array}{l}\text { MIC overrated } \\
\text { by OSNA++ }\end{array}$ & $\begin{array}{l}\text { MIC underrated } \\
\text { by OSNA- }\end{array}$ & $\begin{array}{l}\text { OSNA+ overrated } \\
\text { as MAC }\end{array}$ & $\begin{array}{l}\text { OSNA+ underrated } \\
\text { as ITC/NEG }\end{array}$ \\
\hline Visser $^{24}$ & 7 & 26 & 11 & 20 & 5 & 4 & 2 & 2 & 13 \\
\hline Snook $^{18}$ & 17 & 30 & 12 & 27 & 9 & 1 & 2 & 8 & 10 \\
\hline Feldman ${ }^{27}$ & 19 & 81 & 43 & 50 & 12 & 9 & 22 & 9 & 29 \\
\hline Bernet $^{28}$ & 8 & 14 & 9 & 10 & 5 & 2 & 2 & 1 & 4 \\
\hline
\end{tabular}

ITC, isolated tumour cells/clusters; MAC, macrometastasis (>2 mm); MIC, micrometastasis; NEG, negative by histology; OSNA+, one-step nucleic acid amplification positive with 250-5000

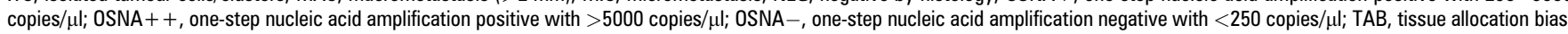


include benign epithelial inclusions ${ }^{28}$ or epithelial cell displacement. Benign epithelial inclusions are very rare, only one has been reported in the series summarised here ${ }^{28}$ and seven were seen in the Milan series consisting of more than 3500 SLNs. ${ }^{31}$ Most of these are small, around the size, volume or cell number of ITC and would most probably remain undetected by OSNA, similar to smaller bits of tumour cells reaching the lymph nodes by artefactual displacement and passive transport. ${ }^{32}$ Although there have been reports of low levels of CK19 expression in the lymph nodes of patients without cancer, ${ }^{33} 34$ this is not supported by other studies, ${ }^{12}$ and low levels of illegitimate CK19 expression are probably overcome by the cut-off value for a positive OSNA test. ${ }^{34}$

\section{OSNA AS THE GOLD STANDARD}

It is well accepted that the more thorough the sampling, the more nodal metastatic deposits are discovered by histology, although most of the lesions detected by extra sectioning and by diminishing the interval between sections analysed belong to the micrometastasis or ITC categories. ${ }^{35}$ No histology protocol can aim at detecting all tumour cells within a lymph node, and the major advantage of a systemic multilevel pathology approach is to achieve a more homogeneous node-negative breast cancer category by excluding occult metastases of a given size (the sectioning interval) as best as possible. ${ }^{36}$

As OSNA can sample the whole of the lymph node portion that is submitted to this test, it is very tempting to propose that OSNA with a good overall performance when compared with histopathology by alternate slice investigation studies (tables 2 and 3 ) is more sensitive than histology and is almost as specific. It could therefore be the gold standard when comparisons are made. Table 4 explores this suggestion.

In this setting, while histology has a good specificity in detecting metastases, its sensitivity is inferior to that of OSNA, which can be explained mainly by the sampling differences, but also by the fact that histopathologists may sometimes miss low volume metastases. ${ }^{37-39}$ If OSNA is more likely to demonstrate all nodal involvement classified as metastases (excluding ITC), it is also evident that the false-negative rate and false-reassurance rate of histology is inferior to that of OSNA (tables 2 and 4). So, if the aim is to find all metastases, OSNA might be a better choice than conventional histology, but this requires some important considerations discussed below.

In fact, some laboratories have introduced OSNA in their daily routine, outside of clinical trials. ${ }^{18} 192840-42$ In contrast to

Table 4 Results of histological and OSNA tests in different validation series, OSNA being the gold standard for comparison

\begin{tabular}{lccccccc}
\hline Reference (first author) & SENS & SPEC & ACC & PPV & NPV & FNR & FRR \\
\hline Tsujimoto $^{12}$ & 95.6 & 99.3 & 98.8 & 95.6 & 99.3 & 4.4 & 0.7 \\
Visser $^{24}$ & 88.4 & 98.9 & 96.8 & 95.3 & 97.1 & 11.6 & 2.9 \\
Schem $^{25}$ & 87.4 & 100 & 95.5 & 100 & 93.4 & 12.6 & 6.6 \\
Tamaki Trial1 $^{26}$ & 86.4 & 99 & 96.8 & 95 & 97.1 & 13.6 & 2.9 \\
Tamaki Trial2 $^{26}$ & 77.8 & 99.4 & 95 & 97.2 & 94.6 & 22.2 & 5.4 \\
Snook $^{18}$ & 86.8 & 98.1 & 95.9 & 91.7 & 96.9 & 13.2 & 3.1 \\
Feldman $^{27}$ & 84.3 & 96.7 & 95.1 & 78.1 & 97.8 & 15.7 & 2.3 \\
Bernet $^{28}$ & 94.3 & 99.3 & 98.3 & 97.1 & 98.6 & 5.7 & 1.4 \\
Le Frere-Belda $^{14}$ & 81 & 98.8 & 96.5 & 91.1 & 97.2 & 19 & 2.8 \\
All & $\mathbf{8 5 . 8}$ & $\mathbf{9 8 . 3}$ & $\mathbf{9 6 . 1}$ & $\mathbf{9 1 . 7}$ & $\mathbf{9 7}$ & $\mathbf{1 4 . 2}$ & $\mathbf{3}$ \\
\hline
\end{tabular}

ACC, accuracy (concordance rate of the two tests); FNR, false-negative rate (falsenegatives/all positives); FRR, false-reassurance rate (false-negatives/all testing negative) NPV, negative predictive value; OSNA, one-step nucleic amplification; PPV, positive predictive value; SENS, sensitivity; SPEC, specificity; values expressed as percentages. alternate slice sampling used in validation studies, whole SLNs have been used for the molecular test, ${ }^{19} 2841$ or the central 1-mm thick layer of the SLN was submitted for histology and the rest of the nodes for OSNA, ${ }^{40}$ which constitutes a good compromise for having as much of the lymph node as possible for a sensitive method giving intraoperative results and still allowing for basic and minimal morphological assessment of larger metastases and non-metastatic changes in the lymph node. Some institutions have adopted the minimum of looking at imprint cytology specimens before homogenisation of the whole SLN. ${ }^{42}$ Because there are places in Europe where legislation requires the submission of surgically removed tissues to histological examination, the splitting of lymph node material to OSNA and histology should also consider legal issues. Certainly, at present, patients should be informed about the basics of the OSNA procedure and give an informed consent.

Positivity rates of $31 / 211(14.7 \%$, after exclusion of the cases labelled as ITC), ${ }^{41} 42 / 197(21.3 \%),{ }^{40} 121 / 531(22.8 \%)^{19}$ and $32 /$ $110(29.1 \%)^{42}$ have been reported outside validation series, whereas a recently published study cited a $28.2 \%$ rate for SLN positivity provided by Sysmex. ${ }^{18}$ Interestingly, the OSNA-based SLN positivity rate in two series was slightly lower than the positivity rate experienced before the introduction of OSNA ( $21 \%$ vs $25 \%$ and $14 \%$ vs $22 \%$, the latter being significantly different on the basis of a $\chi^{2}$ test), although other parameters of the compared groups were similar. ${ }^{40}{ }^{41}$ In another series of patients with similar tumours studied either by detailed histology and immunohistochemistry or by OSNA, the rate of SLN-negative patients was similar for both tests, but OSNA detected more micrometastases (18\% vs $8 \%$ ) and fewer macrometastases (11\% vs $20 \%$ ) than histology, ${ }^{42}$ although the $95 \% \mathrm{CI}$ of these proportions overlap.

Few data are published at present as concerns the rate of nonSLN involvement in patients with SLNs analysed by OSNA only, but the small series by Khaddage et al ${ }^{40}$ reported a proportion of seven of 13 (0.54) for OSNA + + cases and three of $29(0.10)$ for OSNA + cases, in keeping with a quantitative difference reported by histology-based studies. Similar differences were seen in another small series from Turin: five of 12 (0.42) of the cases reported to be macrometastatic by OSNA + + were non-SLN positive, whereas only two of nine (0.22) of the micrometastatic (OSNA + ) cases had further nodes involved at ALND. $^{42}$

\section{Concerns}

Although many papers, including recent ones, ${ }^{1}$ start with the statement that lymph node status is the most relevant single prognostic factor of breast cancer, there are now limitations in establishing real prognostic markers, as most patients receive some kind of systemic adjuvant treatment, and a 'per definitionem' prognostic marker reflects the effect of that factor in patients without systemic treatment. As the most important prognostic marker, lymph node status was a factor with unique importance in guiding the administration of systemic therapy. Currently, lymph node status has decreased in importance both in terms of prognostication and treatment planning. Many patients belong to the lymph node-negative category, and nodal status is just one factor to be considered with less weight and more balancing when considering systemic chemotherapy in breast cancer patients. ${ }^{43} 44$

The introduction of SLN biopsy has resulted in a nodal upstaging rate between $9 \%$ and $47 \%$ due to more scrutiny given to the SLNs. ${ }^{45}$ The wide range in upstaging has been largely attributed to differences in histopathology protocols, ${ }^{17}$ which 
lack standardisation, despite guidelines created with this aim. ${ }^{46}$ Although there are increasing amounts of data suggesting that micrometastases are of prognostic importance, ${ }^{47} 48$ there has also been a proposal suggesting that micrometastases detected in SLN do not have the same bearing on prognosis as micrometastases from older series. ${ }^{49}$ If this proved true and was confirmed by others, it seems that micrometastases should not be looked for in SLN samples, and the general recommendation of identifying possibly all macrometastases ${ }^{36} 50$ would be further supported. In line with this notion, the survival impact of occult metastases in SLNs was found to be minimal and negligible in the NSABP-B32 trial. ${ }^{51}$ Following this reasoning, OSNA would come in the armamentarium as a tool increasing the detection of metastases intraoperatively, but especially increasing the detection rate of micrometastases of lower impact. ${ }^{41}$ It seems that for macrometastases, current practices of frozen sectioning and standard histopathology also result in a high enough and acceptable sensitivity. ${ }^{4}$

OSNA has the advantage of being standardised, therefore interpretation issues are probably less problematical than with histopathology in which distinction between ITC (a node-negative category) and micrometastasis (a node-positive category) was suboptimal. ${ }^{52-55}$ It has been reported that OSNA is sometimes inhibited, and this results in false-negative $(<250$ copies $/ \mu \mathrm{l})$ tests, which can be resolved as positive simply on the basis of producing positive tests $(>250$ copies $/ \mu \mathrm{l})$ in the 1:10 diluted samples, in which the inhibitory material may also be diluted. ${ }^{19}$ The results of such inhibited reactions cannot be unequivocally categorised as OSNA+ or OSNA ++. For sure, a standardised approach such as OSNA better help in clarifying the prognostic role of low volume metastases, although the equivalence between OSNA+/OSNA++ and micrometastasis/(macro)mestastasis is only based on realistic estimates. ${ }^{12}{ }^{14}$ Nodal staging based on histology is a recognised prognosticator, but OSNA-based staging is just an extrapolated one. Although this approach is probably correct, it is not validated. Some have also used a copy number between 100 and $250 / \mu \mathrm{l}$ as an equivalent of ITC, ${ }^{41}$ but the specificity of this type of equivalence is even more difficult to ascertain. The only tumour-node-metastasis category defined for lymph nodes being positive by molecular assays without being positive by histology is $\mathrm{pNO}(\mathrm{mol}+)$ and reflects a subset of the ITC category. ${ }^{15} 16$ This category was introduced within a scenario in which molecular testing was added to histology and/or immunohistochemistry and detected nodal positivity undisclosed by morphological methods. This label is misleading and inadequate for most lesions detected by quantitative molecular assays, for example, metastases labelled as OSNA ++. Such nodal metastases should better be coded as $\mathrm{pN1}(\mathrm{mol}+)$, a category that is currently not listed in the tumour-node-metastasis definitions. ${ }^{56}$

Inherent to the more complete sampling without discarded tissue bits, OSNA seems to be more sensitive to detect metastases of small volume and to detect all metastases above the calibrated detection level set at 250 copies of CK19 mRNA/ $\mu \mathrm{l}$. Many of these low volume metastases would remain occult by histology. Therefore, OSNA has the potential to find all metastases whereas the accepted aim of histology is only to exclude metastases of a given size to make a homogeneous $\mathrm{pN} 0$ category.

OSNA is not an extended histology, it is not even an equivalent of histology, and some of the OSNA-negative/histologypositive mismatches are not explainable by other means than the failure of the technique to detect histologically identified metastases. False-positive OSNA results are also possible, as discussed above, but all of these are supposed to be of very low incidence. Due to the lack of morphology when the whole lymph node is processed for a molecular study, several features considered important in some respects are lost; these include the size, location and pattern of the lymph node metastasis, extracapsular extension of a metastasis and the degree of regression after neoadjuvant therapy. The submission of perinodal fat tissue for histology might be a substitute to check for extracapsular metastasis extension. ${ }^{41}$

Although SLN-negative patients are at very low risk of having further lymph node involvement in their axilla, and OSNAnegative SLNs can be extrapolated to represent SLN-negative patients on the basis of the validation series summarised in this review, a subset of SLN-positive patients is also suspected to derive no benefit of completion ALND, as they have no further metastases. This may be especially true for patients with no suspicious lymph nodes on preoperative ultrasound examination of the axilla, because non-SLN involvement is rare in these patients. ${ }^{57}$ Several predictive models have been built in order to predict the risk of non-SLN involvement in breast cancer patients in general, and also for the subset with micrometastasis, in whom the proportion of second echelon lymph node involvement is rather low, approximately $10-15 \%{ }^{58} 59$ These models, besides data derived from the primary tumour, often consider SLN metastasis size, extracapsular extension, the number or proportion of SLNs involved to build up their nomograms or scoring systems. ${ }^{60} 61$ Although none of the models is perfect, such models are used in practice to advise clinicians and patients. With the introduction of OSNA as the sole evaluation method of SLNs, some of the features would be lost, therefore new models using the data from OSNA should be built up, and this is probably one way in which new research will be initiated.

Technical failure of the OSNA system was also reported in six cases $(1.4 \%)$ in the UK multi-institutional study. ${ }^{18}$ In such cases, should the whole lymph node be used for the molecular assay, no nodal staging could be achieved after homogenisation of the SLN. In such instances, repeat OSNA or an alternative molecular test (from the palette used in the validation series) of the same lysate should be able to replace the initially failed test, but such a scenario has to be planned in advance, and there should be an action plan in order to resolve these issues.

The costs of OSNA and histology are very much site dependent. In the eastern part of Europe histology costs a lot less than in the founding counties of the European Union. Although a cost analysis has recently been published from Spain, and suggested an average saving of over $€ 400$ per patient, ${ }^{62}$ there are places where the introduction of this molecular staging method would result in an increase of at least some parts of the costs. For example, as a non-reimbursed method (eg, in Hungary), it would only produce deficit to the departments using it, at least at the level of direct costs of nodal staging. In terms of second surgeries, not all patients with positive SLN accept reoperations, and not all second surgeries are performed because of a falsenegative intraoperative SLN status; some are indicated because of involved or close margins of the primary tumour. Therefore, a realistic cost analysis should also consider these more complex issues and the OSNA-related publications do not mention such complexity. There are also cases in which OSNA only increases the costs, when it is used irrationally. Naked eye examination can sometimes reveal nodal metastasis and this can certainly more easily be confirmed by a more rapid and cheaper microscopic examination. This selective attitude was probably reflected in a recent Spanish publication. ${ }^{41}$ If technicians or clinicians at the operating room deal with OSNA, as suggested 
by some authors, ${ }^{40}$ this opportunity of faster intraoperative diagnosis at lower cost would be definitely lost. Differences in reimbursement policies and healthcare organisation issues may also act controversially on the costs: intraoperative (BLN assaybased molecular) SLN analysis was estimated to produce nearly $£ 300$ of loss per patient when compared with no SLN biopsy and immediate ALND for all patients. ${ }^{63}$

It must also be remembered that several roads may lead to the same endpoint. For example, ALND seems to be replaceable by radiotherapy. Although the American College of Surgeons Oncology Group Z11 trial has some limitations, it points to the fact that the standard addition of systemic adjuvant treatment and opposing tangentional field whole breast irradiation including a significant part of the axilla may result in similar survivals to ALND in patients with positive SLN treated with breast conservation. ${ }^{64}$ The EORTC 10981-22023 (AMAROS) trial is also exploring the issue of whether axillary radiotherapy can reach the same rates of regional disease control as completion ALND in SLN-positive patients. ${ }^{65}$ So some centres, or some patients disliking the idea of an ALND, may not need intraoperative assessment at all, because the indication for systemic therapy will be based on the final nodal stage reached by permanent section histology, which is a validated method in this respect, even if OSNA may be more precise on the basis of data presented in tables 2 and 4 . There is also a risk of overdiagnosis, in the epidemiological meaning, ie, discovering minute changes (metastases) that would not require action. Treating them the same way as larger metastases would result in overtreatment in most cases. These issues will require longer experience with OSNA-based staging, but for sure, this is to come in the future.

\section{CONCLUSIONS}

OSNA is a technique more and more widely applied to the intraoperative investigation of SLNs in breast cancer patients. ${ }^{18}$ Validation studies published to date are consistent with a reliable quantitative test that allows final decisions related to axillary treatment of patients. Its results can also be applied to replace histology in the prognostication of breast cancer and substitute it in systemic treatment-related decisions, although there are no studies to date about the value of metastases detected by OSNA only, in particular no data are available for low volume micrometastases. As OSNA is implemented in some places, it should be incorporated in the diagnosis and prognostication of breast carcinomas as an alternative test used instead of (or along with) histology. As histology will remain the staging method at most sites, equivalences and extrapolations will be needed, especially for patients migrating between institutions with different staging methods during the delivery of their treatment. In

\section{Take-home messages}

- OSNA is a viable method of intraoperative metastasis detection in sentinel lymph nodes of breast cancer patients.

- OSNA detects more low volume nodal involvement than conventional intraoperative tests or permanent sections in histology, but it is uncertain whether these small metastases require further axillary treatment.

- It is uncertain whether the advantage of OSNA of detecting practically all metastases is clinically more important than the exclusion of metastases greater than micrometastasis which can be reliably done by intraoperative microscopy. keeping with previous wording of the European guidelines, ${ }^{48}$ it seems wise to make a compromise and to give up a bit of staging accuracy and to conserve a bit of morphological assessment, although some centres will use the whole lymph node for staging purposes.

\section{Competing interests None.}

Provenance and peer review Not commissioned; externally peer reviewed.

\section{REFERENCES}

1. Lyman GH, Giuliano AE, Somerfield MR, et al. American Society of Clinical Oncology guideline recommendations for sentinel lymph node biopsy in early-stage breast cancer. J Clin Oncol 2005;23:7703-20.

2. Giuliano AE, Dale PS, Turner RR, et al. Improved axillary staging of breast cancer with sentinel lymphadenectomy. Ann Surg 1995;222:394-401.

3. Tew K, Irving L, Matthews A, et al. Meta-analysis of sentinel node imprint cytology in breast cancer. Br J Surg 2005;92:1068-80.

4. Liu LC, Lang JE, Lu Y, et al. Intraoperative frozen section analysis of sentinel lymph nodes in breast cancer patients. Cancer 2011;117:250-8.

5. Viale G, Bosari S, Mazzarol G, et al. Intraoperative examination of axillary sentinel lymph nodes in breast carcinoma patients. Cancer 1999;85:2433-8.

6. Ku NN. Pathologic examination of sentinel lymph nodes in breast cancer. Surg Oncol Clin North Am 1999;8:469-79.

7. Salem AA, Douglas-Jones AG, Sweetland HM, et al. Intraoperative evaluation of axillary sentinel lymph nodes using touch imprint cytology and immunohistochemistry. Part II. Results. Eur J Surg Oncol 2006;32:484-7.

8. Nahrig JM, Richter T, Kuhn W, et al. Intraoperative examination of sentinel lymph nodes by ultrarapid immunohistochemistry. Breast J 2003:9:277-81.

9. Leikola JP, Tolvonen TS, Krogerus LA, et al. Rapid immunohistochemistry enhances the intraoperative diagnosis of sentinel lymph node metastases in invasive lobular breast carcinoma. Cancer 2005;104:14-19.

10. Choi YJ, Yun HR, Yoo KE, et al. Intraoperative examination of sentinel lymph nodes by ultrarapid immunohistochemistry in breast cancer. Jpn J Clin Oncol 2006;36:489-93

11. Blumencranz $\mathbf{P}$, Whitworth PW, Deck K, et al. Scientific Impact Recognition Award Sentinel node staging for breast cancer: intraoperative molecular pathology overcomes conventional histologic sampling errors. Am J Surg 2007;194:426-32.

12. Tsujimoto $\mathbf{M}$, Nakabayashi K, Yoshidome K, et al. One-step nucleic acid amplification for intraoperative detection of lymph node metastasis in breast cance patients. Clin Cancer Res 2007:13:4807-16.

13. Notomi T, Okayama $\mathrm{H}$, Masubuchi $\mathrm{H}$, et al. Loop-mediated isothermal amplification of DNA. Nucleic Acids Res 2000;28:E63.

14. Le Frère-Belda MA, Bats AS, Gillaizeau F, et al. Diagnostic performance of one-step nucleic acid amplification for intraoperative sentinel node metastasis detection in breast cancer patients. Int J Cancer. Published Online First: 21 July 2011. doi:10.1002/ijc.26291.

15. Sobin L, Gospodarowicz M, Wittekind Ch, eds. UICC TNM Classification of Malignan Tumours, 7th edn. New York: John Wiley and Sons Inc, 2009

16. Edge SB, Byrd DR, Compton CC, et al, eds. AJCC Cancer Staging Handbook: From the AJCC Cancer Staging Manual. New York: Springer, 2009.

17. Cserni G, Amendoeira I, Apostolikas N, et al. Discrepancies in current practice of pathological evaluation of sentinel lymph nodes in breast cancer. Results of a questionnaire-based survey by the European Working Group for Breast Screening Pathology. J Clin Pathol 2004;57:695-701.

18. Snook KL, Layer GT, Jackson PA, et al. Multicentre evaluation of intraoperative molecular analysis of sentinel lymph nodes in breast carcinoma. Br J Surg 2011:98:527-35

19. Osako T, Iwase T, Kimura K, et al. Intraoperative molecular assay for sentinel lymph node metastases in early stage breast cancer: a comparative analysis between onestep nucleic acid amplification whole node assay and routine frozen section histology. Cancer 2011:117(19):4365-74.

20. Smith PA, Harlow SP, Krag DN, et al. Submission of lymph node tissue for ancillary studies decreases the accuracy of conventional breast cancer axillary node staging. Mod Pathol 1999;12:781-5.

21. Cserni G. Mapping metastases in sentinel lymph nodes of breast cancer. Am J Clin Pathol 2000;113:351-4.

22. Diaz LK, Hunt $K$, Ames $F$, et al. Histologic localization of sentinel lymph node metastases in breast cancer. Am J Surg Pathol 2003:27:385-9.

23. Daniele L, Annaratone L, Allia E, et al. Technical limits of comparison of stepsectioning, immunohistochemistry and RT-PCR on breast cancer sentinel nodes: a study on methacarn fixed tissue. J Cell Mol Med 2009;13:4042-50.

24. Visser M, Jiwa M, Horstman A, et al. Intra-operative rapid diagnostic method based on CK19 mRNA expression for the detection of lymph node metastases in breast cancer. Int J Cancer 2008;122:2562-7.

25. Schem C, Maass N, Bauerschlag D0, et al. One-step nucleic acid amplification-a molecular method for the detection of lymph node metastases in breast cancer patients; results of the German study group. Virchows Arch 2009;454:203-10.

26. Tamaki Y, Akiyama F, Iwase T, et al. Molecular detection of lymph node metastases in breast cancer patients: results of a multicenter trial using the one-step nucleic acid amplification assay. Clin Cancer Res 2009;15:2879-84. 
27. Feldman S, Krishnamurthy S, Gillanders W, et al. A novel automated assay for the rapid identification of metastatic breast carcinoma in sentinel lymph nodes. Cancer 2011;117:2599-607.

28. Bernet $\mathbf{L}$, Cano $\mathrm{R}$, Martinez $\mathrm{M}$, et al. Diagnosis of the sentinel lymph node in breast cancer: a reproducible molecular method: a multicentric Spanish study. Histopathology 2011:58:863-9.

29. Chu PG, Weiss LM. Keratin expression in human tissues and neoplasms. Histopathology 2002;40:403-39.

30. Alvarenga CA, Paravidino $\mathrm{Pl}$, Alvarenga $\mathrm{M}$, et al. Expression of CK19 in invasive breast carcinomas of special histological types: implications for the use of one-step nucleic acid amplification. J Clin Pathol 2011;64:493-7.

31. Maiorano E, Mazzarol GM, Pruneri G, et al. Ectopic breast tissue as a possible cause of false-positive axillary sentinel lymph node biopsies. Am J Surg Pathol 2003;27:513-18.

32. Bleiweiss IJ, Nagi CS, Jaffer S. Axillary sentinel lymph nodes can be falsely positive due to iatrogenic displacement and transport of benign epithelial cells in patients with breast carcinoma. J Clin Oncol 2006;24:2013-18.

33. Yun K, Gunn J, Merrie AE, et al. Keratin 19 mRNA is detectable by RT-PCR in lymph nodes of patients without breast cancer. Br J Cancer 1997;76:1112-13

34. Bostick PJ, Chatterjee S, Chi DD, et al. Limitations of specific reverse-transcriptase polymerase chain reaction markers in the detection of metastases in the lymph nodes and blood of breast cancer patients. J Clin Oncol 1998;16:2632-40.

35. Cserni G. Complete step sectioning of axillary sentinel lymph nodes in patients with breast cancer. Analysis of two different step sectioning and immunohistochemistry protocols in 246 patients. J Clin Pathol 2002;55:926-31.

36. Weaver DL. Pathology evaluation of sentinel lymph nodes in breast cancer: protocol recommendations and rationale. Mod Pathol 2010;23:S26-32.

37. Mesker WE, Torrenga $\mathrm{H}$, Sloos WC et al. Supervised automated microscopy increases sensitivity and efficiency of detection of sentinel node micrometastases in patients with breast cancer. J Clin Pathol 2004;57:960-4.

38. Weaver DL, Krag DN, Manna EA, et al. Detection of occult sentinel lymph node micrometastases by immunohistochemistry in breast cancer. An NSABP protocol B-32 quality assurance study. Cancer 2006;107:661-7.

39. Cserni G, Bianchi S, Vezzosi V, et al. The value of cytokeratin immunohistochemistry in the evaluation of axillary sentinel lymph nodes in patients with lobular breast carcinoma. J Clin Pathol 2006:59:518-22.

40. Khaddage A, Berremila SA, Forrest $F$, et al. Implementation of molecular intra-operative assessment of sentinel lymph nodes in breast cancer. Anticancer Res 2011:31:585-90.

41. Laia BV, Marcos MB, Refael CM, et al. Molecular diagnosis of sentinel lymph nodes for breast cancer: one step ahead for standardization. Diagn Mol Pathol 2011;20:18-21.

42. Castellano I, Macri L, Deambrogio C, et al. Reliability of whole sentinel lymph node analysis by One-Step Nucleic Acid Amplification for intraoperative diagnosis of breast cancer metastases. Ann Surg 2012;255(2):334-42.

43. Goldhirsch A, Ingle JN, Gelber RD, et al. Thresholds for therapies: highlights of the St Gallen International Expert Consensus on the primary therapy of early breast cancer 2009. Ann Oncol 2009;20:1319-29.

44. National Comprehensive Cancer Network. Clinical practice guidelines in onology -Breast cancer. Verion 2.2011. http://www.nccn.org/professionals/ physician_gls/pdf/breast.pdf (accessed 3 Jul 2011).

45. Cserni G, Amendoeira I, Apostolikas N, et al. Pathological work-up of sentinel lymph nodes in breast cancer. Review of current data to be considered for the formulation of guidelines. Eur J Cancer 2003;39:1654-67.

46. Cserni G. Histopathologic examination of the sentinel lymph nodes. Breast $J$ 2006:12(Suppl 2):S152-6.
47. Dowlatshahi K, Fan M, Snider HC, et al. Lymph node micrometastases from breas carcinoma: reviewing the dilemma. Cancer 1997;80:1188-97.

48. Truong PT, Vinh-Hung V, Cserni G, et al. The number of positive nodes and the ratio of positive to excised nodes are significant predictors of survival in women with micrometastatic node-positive breast cancer. Eur J Cancer 2008;44:1670-7.

49. Montagna E, Viale G, Rotmensz N, et al. Minimal axillary lymph node involvement in breast cancer has different prognostic implications according to the staging procedure. Breast Cancer Res Treat 2009;118:385-94.

50. Amendoeira I, Apostolikas N, Bellocq JP, et al: EC Working Group on Breast Screening Pathology. Quality assurance guidelines for pathology. In: Perry N Broeders $\mathrm{M}$, de Wolf $\mathrm{C}$, et al, eds. European Guidelines for Quality Assurance in Breast Cancer Screening and Diagnosis, 4th edn. Luxemburg: European Comission, 2006:219-311.

51. Weaver DL, Ashikaga T, Krag DN, et al. Effect of occult metastases on survival in node-negative breast cancer. N Engl J Med 2011:364:412-21.

52. Cserni G, Bianchi S, Boecker W, et al. Improving the reproducibility of diagnosing micrometastases and isolated tumour cells. Cancer 2004;103:358-67.

53. De Mascarel I, MacGrogan G, Debled M, et al. Distinction between isolated tumo cells and micrometastases in breast cancer: is it reliable and useful? Cancer 2008:112:1672-8.

54. Turner RR, Weaver DL, Cserni G, et al. Nodal stage classification for breast carcinoma: improving interobserver reproducibility through standardized histologic criteria and image-based training. J Clin Oncol 2008;26:258-63.

55. Cserni G, Amendoeira I, Bianchi S, et al. Distinction of isolated tumour cells and micrometastasis in lymph nodes of breast cancer patients according to the new Tumour Node Metastasis (TNM) definitions. Eur J Cancer 2011:47:887-94.

56. Cserni G. The current TNM classification of breast carcinomas: controversial issues in early breast cancer. MEMO 2011:4:144-8.

57. Zgajnar J, Hocevar M, Podkrajsek M, et al. Patients with preoperatively ultrasonically uninvolved axillary lymph nodes: a distinct subgroup of early breast cancer patients. Breast Cancer Res Treat 2006:97:293-9.

58. Cserni G, Gregori D, Merletti F, et al. Non-sentinel node metastases associated with micrometastatic sentinel nodes in breast cancer: metaanalysis of 25 studies. $\mathrm{Br} \mathrm{J}$ Surg 2004;91:1245-52.

59. Houvenaeghel G, Nos C, Giard S, et al. A nomogram predictive of non-sentine lymph node involvement in breast cancer patients with a sentinel lymph node micrometastasis. Eur J Surg Oncol 2009;35:690-5.

60. Degnim AC, Griffith KA, Sabel MS, et al. Clinicopathologic features of metastasis in nonsentinel lymph nodes of breast carcinoma patients. Cancer 2003: 98:2307-15

61. Van la Parra RF, Peer PG, Ernst MF, et al. Meta-analysis of predictive factors for non-sentinel lymph node metastases in breast cancer patients with a positive SLN. Eur J Surg Oncol 2011;37:290-9.

62. Guillen-Paredes MP, Carrasco-Gonzalez L, Chaves-Benito A, et al. [One-step nucleic acid amplification (OSNA) assay for sentinel lymph node metastases as an alternative to conventional postoperative histology in breast cancer: A cost-benefit analysis.] Cir Esp 2011;89(7):456-62.

63. Cutress RI, McDowell A, Gabriel FG, et al. Observational and cost analysis of the implementation of breast cancer sentinel node intraoperative molecular diagnosis. $J$ Clin Pathol 2010:63:522-9.

64. Giuliano AE, McCall L, Beitsch $P$, et al. Locoregional recurrence after sentinel lymph node dissection with or without axillary dissection in patients with sentinel lymph node metastasis. Ann Surg 2010;252:426-33.

65. Straver ME, Meijnen P, van Tienhoven G, et al. Sentinel node identification rate and nodal involvement in the EORTC 10981-22023 AMAROS trial. Ann Surg Oncol 2010:17:1854-61. 